\title{
Traditional Chinese Medicinal Syndromes and Treatment in Colorectal Cancer
}

\author{
Shan Deng, Bing Hu* , Hong-Mei An \\ Department of Oncology and Institute of Traditional Chinese Medicine in Oncology, Longhua Hospital, Shanghai University of Tra- \\ ditional Chinese Medicine, Shanghai, China. \\ Email: *beearhu@hotmail.com
}

Received September $16^{\text {th }}$, 2012; revised October $15^{\text {th }}, 2012$; accepted October $24^{\text {th }}, 2012$

\begin{abstract}
Colorectal cancer remains the third most common malignancy worldwide. According to the principles of Traditional Chinese Medicine (TCM), colorectal cancer is related to spleen-deficiency, damp-heat, and toxicity accumulation. Traditional Chinese medicine has been confirmed to effectively reduce toxic side effects and enhance curative effects of chemotherapy, palliate clinical syndrome, prevent recurrence and metastasis, improve quality of life and immune function, and prolong survival time in colorectal cancer. However, TCM theoretical and syndrome differentiation study has lagged far behind due to progression of therapy model in colorectal cancer. As a type of drug treatment, there are some common biological basis between chemotherapy, targeted therapy and anti-cancer herb therapy in colorectal cancer, such as apoptosis, cell senescence and autophagy. There are growing needs to explore the effect mechanism and compatibility of anti-cancer herbs, to further enhance the efficacy of TCM treatment in colorectal cancer.
\end{abstract}

Keywords: Colorectal Cancer; Syndrome Differentiation; Traditional Chinese Medicinal Treatment; Anti-Cancer Herb

\section{Introduction}

Colorectal cancer remains the third most commonly diagnosed cancer in males and the second in females worldwide. The incidence of colorectal cancer is increasing due to smoking, lack physical activities, overweight and obesity, red and processed meat consumption, and excessive alcohol consumption [1]. The current treatment of colorectal cancer mainly depends on surgery, chemotherapy, radiotherapy and targeted therapy. In China, Traditional Chinese Medicine (TCM) plays an important role in the treatment of colorectal cancer. The description of colorectal cancer in TCM can be traced back to the era of Huang-Di's Canon of Internal Medicine (400 B.C.). From TCM perspective, the carcinogenesis of colorectal cancer is related to the $\mathrm{Pi}-\mathrm{Xu}$ (spleen-Qi deficiency), Shi-Re (damp-heat) and Du-Ju (toxicity accumulation). Accumulated studies have showed that TCM may help to reduce the side effects and enhance the curative effects of chemotherapy, palliate clinical syndrome, prevent recurrence and metastasis, prolong survival time and improve the quality of life in colorectal cancer.

\section{Syndrome Differentiation in Colorectal Cancer}

TCM syndrome (ZHENG) is a kind of pathological

\footnotetext{
"Corresponding author.
}

status that integrated the information of TCM pathogenesis and clinical manifestation. Syndrome is the basis of individualized TCM treatment (Syndrome Based Differential Treatment). The clinical manifestation of colorectal cancer is complex and different patients may present different TCM syndromes. Based on clinical epidemiology study, Wang et al. reported that the Shi-Re (dampheat) is the most frequent observed syndrome in colorectal cancer, followed by Pi-Wei-Qi-Xu (spleen and stomach deficiency), Qi-Xue-LiangXu (both Qi and blood deficiency), and Gan-Shen-Yin-Xu (liver and kidney deficiency). Shi-Re (Damp-heat), the most common basic syndrome, always accompanied by other syndromes [2]. Lin et al. found that the descending order of appearance frequency of TCM syndromes in colorectal cancer is $\mathrm{Pi}-\mathrm{Xu}$ (spleen deficiency), Qi-Xu (Qi deficiency), Yin-Xu (Yin deficiency), Xue-Xu (blood deficiency), Tan-Shi (phlegm dampness), Re-Du (heat-toxicity), Qi-Zhi (Qi stagnation), Yang-Xu (Yang deficiency) and Xue-Yu (blood stasis). In addition, the survival time of Yin-Xu (Yin deficiency) patients is shorter than that of non-Yin deficiency patients, and the survival time of simple Qi deficiency syndrome patients is longer than that of non-simple Qi deficiency syndrome patients. However, the survival time of pure spleen deficiency syndrome patients has no difference from that of nonpure spleen deficiency syndrome patients. Yin deficiency 
and simple Qi deficiency could be considered as predictors of colorectal cancer prognosis [3].

By analyzing literature published in the past decade, Zhao et al. found that the common syndromes in colorectal cancer, including Pi-Shen-Yang-Xu (spleen-kidney Yang deficiency), Shi-Re (damp-heat), Gan-Shen-YinXu (liver-kidney Yin deficiency), Qi-Xu-Liang-Kui (Qi and blood deficiency), Yu-Du (stasis-toxin), Pi-Xu (spleen deficiency), Qi-Zhi-Xue-Yi (Qi stagnation and blood stasis) and Shi-Re-Yun-Du (damp-heat and accumulated toxin). Among them, spleen deficiency plays an important role in the pathogenesis of colorectal cancer [4]. By statistics relevant literatures about TCM syndromes of colorectal cancer in the past 30 years, Deng et al. found that among the deficiency syndromes of colorectal cancer, Pi-Shen-Yang-Xu (spleen-kidney Yang deficiency) and Qi-Xue-Liang-Xu (Qi-blood deficiency) are the most common syndromes. For sthenia syndromes, Shi-Re (dampheat) and Yu-Du (stasis-toxin) are frequently observed. The top five syndromes are Pi-Shen-Yang-Xu (spleenkidney Yang deficiency), Qi-Xue-Liang-Xu (Qi-blood deficiency), Gan-Shen-Yin-Xu (liver-kidney Yin deficiency), Shi-Re (damp-heat syndrome) and Yu-Du (stasis-toxin) [5].

In different stages of colorectal cancer, the syndromes may be different. Jia et al. reported that TCM syndromes of colorectal cancer mainly include Qi-Zhi-Xue-Yu (Qi stagnation and blood stasis), Gan-Shen-Yin-Xu (liverkidney Yin deficiency), Shi-Du (damp-heat and toxicity accumulation), Qi-Xue-Liang-Xu (Qi and blood deficiency) and Pi-Shen-Yang-Xu (spleen-kidney Yang deficiency). Among them, Shi-Du (damp-heat and toxicity accumulation) is commonly observed in Dukes A and B stage, Qi-Xue-Liang-Xu (Qi and blood deficiency) is common in Dukes B stage, Qi-Zhi-Xue-Yu (Qi stagnation and blood stasis) and Gan-Shen-Yin-Xu (liver-kidney Yin deficiency) are frequently observed in Dukes C stages. Compared with other syndromes, Qi-Zhi-Xue$\mathrm{Yu}$ (stagnation and blood stasis) and Gan-Shen-Yin-Xu (liver-kidney Yin deficiency) are relatively late than ShiDu (damp-heat and toxicity accumulation) and Qi-XueLiang-Xu (Qi and blood deficiency) [6]. Zhao et al. showed that TCM syndromes of colorectal cancer in adjuvant therapy period includes $\mathrm{Pi}-\mathrm{Xu}-\mathrm{Shi}$-Zu (spleen deficiency and retention of dampness), Qi-Xue-Liang-Xu (Qi-blood deficiency, Gan-Shen-Yin-Xu (liver-kidney Yin deficiency), Shi-Re (damp-heat) and Xue-Yu (blood stasis) [7].

Different treatments may influence the clinical syndromes of colorectal cancer. Wang et al. reported that the major syndrome of colorectal cancer before operation is $\mathrm{Yu}$-Du (stasis-toxin). With the development of colorectal cancer, the proportion of Shi-Re (damp-heat) gradually increases and the deficiency syndrome gradually occurs.
The major syndrome of colorectal cancer after operation is mixed sthenia and asthenia syndrome, but in 3 months after operation, the principal syndrome is mainly sthenia syndrome. In the early stage, Shi-Re (damp-heat) is the most frequently observed sthenia syndrome. One week after operation, Yu-Du (stasis-toxin) gradually becomes the dominant syndrome. The accompanied syndrome in the early stage of post-operation is asthenia syndrome especially Qi deficiency, but decreases after one week [8]. In addition, chemotherapy can cause the deficiency of Zheng Qi (the vital energy) and asthenia syndromes increases after chemotherapy in colorectal cancer [9]. At the same time, syndromes are also related to the side effects of chemotherapy. Xu et al. found, in advanced colorectal cancer, severe side effects of chemotherapy frequently occurred in patients with Pi-Shen-Yang-Xu (spleen-kidney Yang deficiency) and Oi-Xue-Liang-Xu (Qi-blood deficiency) syndrome, and suggested that TCM treatment should give priority to strengthen healthy energy during chemotherapy in advanced colorectal cancer [10].

Although the result of different reports is not consistent with each other, but they all indicate that colorectal cancer is closely related to Pi-Xu (spleen deficiency), Shi-Re (heat-damp), and Du-Ju (toxicity accumulation). The syndrome differentiation of colorectal cancer still needs further studies in consideration of clinical manifestation, different disease and treatment stage, and clinical curative effect.

\section{Clinical Study of TCM Treatment in Colorectal Cancer}

Accumulated clinical studies showed that TCM is effective in colorectal cancer in certain respects, such as reducing side effects and enhancing curative effects of chemotherapy, ameliorating clinical syndromes, preventing recurrence and metastasis, improving quality of life and immune function, and prolonging survival time in colorectal cancer patients (Table 1).

In reducing side effects and enhancing curative effect of chemotherapy, Liu et al. showed that Pi-Shen Decoction may decrease the systemic reaction and digestive tract reaction caused by MFA regiment chemotherapy, and prolong survival time in patients with colorectal cancer [11]. Mok et al. indicated that TCM can significantly ameliorate chemotherapy caused nausea [12]. Through 3-year observation on 120 cases of metastatic colorectal cancer patients, Cao et al. found that the combined treatment of Yi-Qi-Zhu-Yu Decoction and FOLFOX4 regiment can effectively prolong the overall survival time and decrease the side effects of chemotherapy [13]. The studies by Tao et al. showed that treatment based on TCM syndrome differentiation can extend the long-term 
Table 1. Clinical study on TCM treatment in colorectal cancer.

\begin{tabular}{|c|c|c|c|}
\hline Name & Herbs & Effects & Ref. \\
\hline Pi-Shen Decoction & $\begin{array}{l}\text { Astragalus memeranaceus (Huang-Qi), Codonopsis pilosula } \\
\text { (Dang-Shen), Atractylodes macrocephala Koidz. (Bai-Shu), etc. }\end{array}$ & $\begin{array}{l}\text { Reduce the side effects of } \\
\text { chemotherapy, prolong survival }\end{array}$ & [11] \\
\hline CHM & $\begin{array}{l}\text { Scrophularia ningpoensis Hemsl. (Xuan-Shen), Plantago asiatica L. } \\
\text { (Che-Qian-Zi), Lysimachia christinae Hance (Jin-Qian-Cao), } \\
\text { Lygodium japonicum (Thunb.) Sw.(Hai-Jin-Sha), Kochia scoparia } \\
\text { (L.) Schrad.(Di-Fu-Zi), Dianthus superbus L.(Qu-Mai), Areca } \\
\text { catechu L. (Da-Fu-Pi), Aconitum carmichaeli Debx. (Chuan-Wu), }\end{array}$ & $\begin{array}{l}\text { Alleviate chemotherapy induced } \\
\text { nausea }\end{array}$ & {$[12]$} \\
\hline
\end{tabular}

Yi-Qi-Zhu-Yu Decoction $\quad$ tetrandra (Fang-Ji), Curcuma phaeocaulis Val. (Er-Shu), Astragalus memeranaceus (Fisch.) Bge. (Huang-Qi), Codonopsis pilosula (Franch.) Nannf. (Dang-Shen), Atractylodes lancea (Cang-Shu), etc.

Panax ginseng (Ren-Shen), Atractylodes macrocephala(Bai-Shu),

TCM Syndrome Differential Poria cocos (Fu-Ling), Citrus reticulata Blanco (Chen-Pi), Treatment Glycyrrhiza uralensis(Gan-Cao), Smilax China L. (Ba-Qia), the roots

Glycyrrhiza uralensis(Gan-Cao), Smilax China L. (Ba-Qia), the roots
of Actinidia arguta (Teng-Li-Gen), ect.
Astragalus memeranaceus (Huang-Qi), Codonopsis pilosula
(Dang-Shen), Coix Seed (Yi-Yi-Ren), Atractylodes macrocephala
(Bai-Shu), Poria cocos (Fu-Ling), Citrus reticulata Blanco(Chen-Pi),
Aucklandia lappa Decne. (Mu-Xiang), Oldenlandia diffusa
(Bai-Hua-She-She-Cao), Scutellaria barbata (Ban-Zhi-Lian),
Taraxacum mongolicum (Pu-Gong-Yin), Cynanchum
paniculatum (Xu-Chang-Qin).
Codonopsis pilosula (Dang-Shen), Astragalus memeranaceus
(Huang-Qi), Atractylodes macrocephala (Bai-Shu), Poria cocos

Reduce the side effects of chemotherapy, improve overall survival

Jian-Pi-Yi-Qi Decoction (Fu-Ling), Coix Seed (Yi-Yi-Ren), Amomum villosum Lour. (Sha-Ren), Pinellia ternate (Ban-Xia), Sparganium stoloniferum (San-Leng), Curcuma phaeocaulis Val. (Er-Shu).

Improve the prognosis, reduce recurrence and metastasis, prolong disease-free survival

Dendrobium (Shi-Hu), Atractylodes lancea (Cang-Shu), Coix Seed (YI-Yi-Ren), Pinellia ternata (Ban-Xia), Dioscorea opposita Thunb. Reduce the side effects of Fu-Pi-Yi-Wei Decoction (Shan-Yao), Poria cocos (Fu-Ling), Amomum compactum Soland. Ex Maton (Dou-Kou), Gynostemma pentaphyllum (Jiao-Gu-Lan), Paeonia lactiflora (Bai-Shao),

Agastache rugosa (Huo-Xiang). Improve quality of life Improve the quality of life, reduce the side effects of chemotherapy

Astragalus memeranaceus (Huang-Qi), Codonopsis pilosula (Dang-Shen), Atractylodes macrocephala (Bai-Shu), Poria cocos (Fu-Ling), Coix Seed (YI-Yi-Ren), Smilax China L. (Ba-Qia),

$\begin{array}{ll}\text { Jian-Pi-Xiao-Liu Decoction } & \begin{array}{l}\text { Curcuma phaeocaulis Val. (Er-Shu), Curcuma wenyujin (Yu-Jin), } \\ \text { Smilax glabra Roxb. (Tu-Fu-Ling), Scolopendra (Wu-Gong), Arca }\end{array}\end{array}$ chemotherapy, improve quality of life and immune function of patients, increase the effective of chemotherapy

Reduce recurrence and metastasis subcrenata Lischke (Wa-Leng-Zi), Polygonatum sibiricum (Huang-Jin), Cornus officinalis (Shan-Yu-Rou), Epimedium brevicornum (Xian-Lin-Pi), Cuscuta chinensis. (Tu-Si-Zi).

Panax ginseng (Ren-Shen), Poria cocos (Fu-Ling), Atractylodes macrocephala (Bai-Shu), Glycyrrhiza uralensis (Gan-Cao), Fu-Zheng Capsule $\quad$ Myristica fragrans (Rou-Dou-Kou), Citrus reticulata Blanco (Chen-Pi), Aucklandia lappa Decne. (Mu-Xiang), Hordeum vulgare

Reduce recurrence and metastasis, improve the quality of life

L. (Mai-Ya), Gallus gallus domesticus Brisson (Ji-Nei-Jin), etc.

Croton tiglium L. (Ba-Dou), Evodia rutaecarpa (Wu-Zhu-Yu), Zingiber officinale (Gan-Jiang), Cinnamomum cassia (Rou-Gui), Aconitum carmichaeli Debx. (Chuan-Wu), Pinellia ternata (Ban-Xia), Citrus reticulata Blanco (Chen-Pi), etc.

Reduce recurrence and metastasis, improve the quality of life

Panax ginseng (Ren-Shen), Atractylodes macrocephala (Bai-Shu),

Si-Jun-Zi Decoction,

Si-Jun-Zi Decoction,
Chai-Hu-Shu-Gan Decoction, Poria cocos (Fu-Ling), Citrus reticulata Blanco (Chen-Pi), Ba-Zhen Decoction Rehmannia glutinosa. (Shu-Di), ect. 


\begin{tabular}{|c|c|c|c|}
\hline Yi-Qi -Jie-Du Decoction & $\begin{array}{l}\text { Codonopsis pilosula (Dang-Shen), Astragalus memeranaceus } \\
\text { (Huang-Qi), Atractylodes macrocephala (Bai-Shu), Poria cocos } \\
\text { (Fu-Ling), Glycyrrhiza uralensis (Gan-Cao), Citrus aurantium L. } \\
\text { (Zhi-Ke), Citrus reticulata Blanco (Chen-Pi), Curcuma phaeocaulis } \\
\text { Val. (Er-Shu), Oldenlandia diffusa (Bai-Hua-She-She-Cao), } \\
\text { Scutellaria barbata (Ban-Zhi-Lian), the roots of Actinidia arguta } \\
\text { (Teng Li-Gen), Sarcandra glabra. (Zhong-Jie-Feng ), } \\
\text { Angelica Sinensis (Dang-Gui). }\end{array}$ & $\begin{array}{l}\text { Control the progression of the disease, } \\
\text { improve quality of life }\end{array}$ & {$[21]$} \\
\hline Zhao’s No. three Decoction & $\begin{array}{l}\text { Codonopsis pilosula (Dang-Shen), Polyporus umbellatus } \\
\text { (Zhu-Ling), Coix Seed (Yi-Yi-Ren), Atractylodes } \\
\text { macrocephala (Bai-Shu), Poria cocos (Fu-Ling), Pinellia } \\
\text { ternate (Ban-Xia), Citrus reticulata Blanco (Chen-Pi), the leaves of } \\
\text { Eriobotrya japonica (Pi-Pa-Ye), etc. }\end{array}$ & $\begin{array}{l}\text { Control the progression of the disease, } \\
\text { improve quality of life and survival }\end{array}$ & {$[22]$} \\
\hline Jian-Pi Decoction & $\begin{array}{l}\text { ria cocos (Fu-Ling), Glycyrrhiza uralensis (Gan-Cao), Fritillaria } \\
\text { thunbergii Miq. (Zhe-Bei-Mu), Platycodon grandiflorus (Jie-Geng), } \\
\text { Coix Seed (Yi-Yi-Ren), Curcuma phaeocaulis Val. (Er-Shu), Ficus } \\
\text { pumila L. (Mu-Man-Tou). }\end{array}$ & $\begin{array}{l}\text { Stable tumor focus, enhance immune } \\
\text { function, improve quality of life }\end{array}$ & {$[23]$} \\
\hline Ai-Di injection & $\begin{array}{l}\text { Panax ginseng (Ren-Shen), Astragalus memeranaceus. (Huang Qi), } \\
\text { Acanthopanax senticosus (Ci-Wu-Jia), Mylabris phalerata } \\
\text { Pallas (Ban-Mao), etc. }\end{array}$ & $\begin{array}{l}\text { Enhance immune function, reduce the } \\
\text { side effects of chemotherapy, improve } \\
\text { quality of life and survival }\end{array}$ & {$[24-26$} \\
\hline Kang-Ai injection & $\begin{array}{l}\text { Astragalus memeranaceus (Fisch.) Bge. (Huang-Qi), Panax ginseng } \\
\text { (Ren-Shen), Oxymatrine. }\end{array}$ & $\begin{array}{l}\text { Improve quality of life, reduce the } \\
\text { side effects of chemotherapy }\end{array}$ & {$[30]$} \\
\hline Shen-Qi-Fu-Zheng injection & $\begin{array}{l}\text { Astragalus memeranaceus (Huang-Qi), Codonopsis pilosula } \\
\text { (Dang-Shen). }\end{array}$ & $\begin{array}{l}\text { Enhance immune function, improve } \\
\text { quality of life and survival, reduce the } \\
\text { side effects of chemotherapy, improve } \\
\text { the efficacy of chemotherapy }\end{array}$ & {$[31,32]$} \\
\hline Huai-Er Granule & Huaier mycoplasm. & $\begin{array}{l}\text { Enhance immune function, reduce the } \\
\text { side effects of chemotherapy, improve } \\
\text { hematopoietic function }\end{array}$ & {$[33,34]$} \\
\hline Astragalus injection & Astragalus memeranaceus (Fisch.) Bge. (Huang-Qi). & $\begin{array}{l}\text { Enhance immune function, improve } \\
\text { the quality of life, reduce the side } \\
\text { effects of chemotherapy }\end{array}$ & [37] \\
\hline
\end{tabular}

survival time in postoperative patients with colorectal cancer [14].

In improving quality of life, Huang et al. reported that the combined treatment of Jian-Qi-Jie-Du Decoction and FOLFOX-4 regiment can effectively improve clinical syndromes and quality of life in patients with colorectal cancer [15]. Liu et al. demonstrated that combined treatment with Jian-Pi-Yi-Qi herbs may improve quality of life and reduce the side effects of chemotherapy in patients with advanced colorectal cancer [16]. Wu et al. found that Fu-Pi-Yi-Wei Decoction can effectively improve quality of life and enhance immune function in postoperative patients with colorectal cancer [17].

In preventing recurrence and metastasis, Ma et al. showed that Jian-Pi-Xiao-Liu Decoction may effectively decrease the recurrence and metastasis of colorectal cancer [18]. Luo et al. found that Fu-Zheng Capsule and Qu-Xie Capsule have a better performance in the reduction of recurrence and metastasis, and improvement of quality of life in the subsequent consolidation therapy in stage II and III colorectal cancer after radical resection [19]. In 222 elder patients with stage II and III colorectal cancer, Yang found that TCM treatment can greatly reduce the possibility of recurrence on the basis of conventional chemotherapy [20].

For advanced colorectal cancer treatment, Xiong et al. reported Yi-Qi-Qing-Du Decoction combined with Cinobufacini injection and sodium cantharidinate can improve quality of life and control tumor focus in advanced colorectal cancer [21]. You et al. found that Zhao's No. Three Decoction may alleviate clinical syndromes, improve quality of life, stabilize tumor focus, control cancer progression and prolong survival time in patients with advanced colorectal cancer [22]. Yin et al. showed that the combined treatment of spleen-strengthening herbs and chemotherapy has obvious effects on stabilizing tumor focus, improving quality of life and immune function in advanced colorectal cancer [23]. 
In addition to herb decoction therapy, Chinese patent drug also plays an important role in colorectal cancer treatment. The common Chinese patent drugs used in colorectal cancer include Ai-Di injection, Matrine injection, Kang-Ai injection, Shen-Qi-Fu-Zheng injection, Huai-Er granule, Kang-Lai-Te injection, Astragalus injection and others. These Chinese patent drugs have been proved to have a sound adjuvant therapeutic effect in the colorectal cancer.

Ai-Di injection can enhance the curative effect of chemotherapy, reduce side effects of chemotherapy to a certain extent, improve the quality of life and immune function, and extend survival time in colorectal cancer patients [24-26]. Matrine injection can reduce the side effects of chemotherapy, strengthen immune function and improve quality of life in colorectal cancer patients. In improving quality of life, Matrine injection has a better performance in colorectal cancer patients with Shi-Re (damp-heat) and Qi-Zhi-Xue-Yu (Qi stagnation and blood stasis) syndrome [27-29]. Kang-Ai injection may obviously improve quality of life and reduce the side effects of chemotherapy in patients with advanced colorectal cancer [30].

Shen-Qi-Fu-Zheng injection can reduce the toxic and side effects and improve the curative effect of chemotherapy, reduce the chemotherapy influence on the immune function, improve quality of life and prolong the survival time in colorectal cancer patients [31,32]. HuaiEr granule can reduce the toxicity of chemotherapy and improve hemopoietic function as well as the immune function in colorectal cancer patients [33,34]. Kang-LaiTe combined with chemotherapy can improve the efficiency and reduce the side effect of chemotherapy, and improve quality of life and immune function in colorectal cancer patients $[35,36]$. Astragalus injection may relieve clinical syndromes, improve quality of life, and synergize with Interleukin-2 to improve immune function in postoperative colorectal cancer patients [37]. Astragalus polysaccharide can effectively reduce the incidence and decline degree of leukocytopenia caused by chemotherapy [38].

\section{Therapeutic Mechanisms of TCM against Colorectal Cancer}

Current pharmacotherapy, such as chemotherapy, targeted therapy act on cancer cells mainly through cell apoptosis, cell senescence and autophagy and other mechanisms [39-41].

Apoptosis has been recognized as major anti-cancer mechanism of pharmacotherapy. It has been confirmed that Curcuma aromatica and Cantharidin promoted apoptosis and G2/M cell cycle arrest in colorectal cancer cells, and related to caspases activation and cell cycle regulatory protein [42,43]. Spatholobus suberectus promoted apoptosis and G2/M cell cycle arrest in colon cancer, and associated with DNA damage and Chk1/Chk2 phosphorylation [44]. Houttuynia cordata Thunb and Hedyotis diffusa Willd extract induced cell apoptosis in colon cancer cells $[45,46]$. Curcumin decreased colon cancer growth, associated with proteasome inhibition, proliferation suppression, and apoptosis induction in tumor tissues [47]. Other herbs or herbal components, such as Melia toosendan fruit, Hedyotis diffusa Willd, DYZ-2-90, and 4,7-Dimethoxy-5-Methyl-1,3-Benzodioxole also showed apoptosis inducing effects in colorectal cancer cells [4851].

Autophagy, Type II programmed cell death, an evolutionarily conserved self-defense mechanism in which organelles and proteins are sequestered and subsequently degraded through fusion with lysosomes, has been recognized as a target for cancer treatment [41,52]. Bufalin, a component from Venenum Bufonis, induced autophagy-mediated cell death in human colon cancer cells through reactive oxygen species generation and JNK activation [53]. Triterpenes, an ingredient from Ganoderma Lucidum, may inhibit cell proliferation, cell cycle arrest in G0/G1 phase, and autophage by downregulation of expression and phosphorylation of p38 MAPK, upregulation Beclin-1 and LC-3 expression in HT-29 colon cancer [54].

Cell senescence, a state of stable irreversible cell cycle arrest provoked by a variety of stimuli, also contributes to herbal therapeutic response $[40,55,56]$. We have demonstrated that Teng-Long-Bu-Zhong Decoction, a Chinese herb formula, may induce LS-174-T colon cancer cell senescence as indicated by typical large and flatten senescent morphology, positive senescence-associated $\beta$-galactosidase staining and G0/G1 phase cell cycle arrest, and associated with upregulation of p21 and p16 expression and downregulation RB phosphorylation [57].

In prevention cancer metastasis, Norcantharidin may downregulate MMP-9 expression by inhibiting Sp1 transcriptional activity in colorectal cancer CT26 cells [58]. Berberine can inhibit colon cancer cell migration via AMP-activated protein kinase-mediated downregulation of integrin $\beta 1$ signaling [59]. Dauricine inhibited cell invasion in colon cancer cells and related to NF- $\kappa \mathrm{B}$ signaling pathway [60]. Triptolide may inhibit colon cancer cells migration by downregulating VEGF, COX-2, thrombin receptor, CXCR4, TNF receptors, and TGF- $\beta$ receptors [61].

Some herbs can also enhance therapeutic effects of chemotherapy or other therapy. In mismatch repair defect cells, He et al. found that Honokiol can enhance the anti-cancer effect of $\gamma$-irradiation treatment, activate caspase-3, promote cell apoptosis and may relevant to expression of cyclin A1 and D1 and increased phosphory- 
lated p53 [62]. Taiwanofungus camphoratus (Antrodia camphorata) may enhance the cytotoxic effects of Amphotericin B [63]. Tetrandrine and Notoginseng may enhance anti-cancer effects of 5 -Fu in colon cancer cells $[64,65]$.

In prevention pathogenesis of colorectal cancer, adlay bran ethanol extract and residue may inhibit the number of crypt foci premalignant lesion, modify their mucin composition and present a dose-dependent manner [55]. Cistanche deserticola significantly reduced the number of mucosal hyperplasia in Tgfb1Rag2 null mice and correlated with significant stimulation of the immune system, suggested the potential to prevent intestinal inflammation disorders including colorectal cancer [56]. Triptolide may inhibit the colitis-related carcinogenesis of colorectal cancer through downregulation of Rac1 and the JAK/STAT3 pathway [57].

Current studies suggested, besides conventionally improving clinical syndromes, anti-cancer effects of TCM against colorectal cancer and the conventional chemotherapy drugs share common biological basis (Table 2). Related explores will provide new insight into integrated treatment with TCM and western medicine for colorectal cancer.

Table 2. Effects and target of Chinese herb against colorectal cancer.

\begin{tabular}{|c|c|c|c|}
\hline Herb/Compound & Effects & Molecular targets & Ref. \\
\hline Curcuma aromatica & Apotoisis, G2/M arrest & Caspases, Cyclin B1, CDK1 & {$[42]$} \\
\hline Cantharidin & Apotoisis, G2/M arrest & $\begin{array}{c}\text { Caspases, Cyclin A, Cyclin B, CDK1, CHK1, p21, } \\
\text { Fas/CD95, Bax/Bcl-2 }\end{array}$ & [43] \\
\hline Spatholobus suberectus & Apotoisis, G2/M arrest & Chk1, Chk2 & {$[44]$} \\
\hline Houttuynia cordata Thunb & Apotoisis & Reactive oxygen species, Caspases, Bax/Bcl-2 & [45] \\
\hline Hedyotis diffusa Willd & Apotoisis & Caspases, Bax/Bcl-2 & {$[46]$} \\
\hline Curcumin & Apotoisis & Proteasome & [47] \\
\hline Melia toosendan fruit & Apoptosis & Caspse-9, Caspase-3 & {$[48]$} \\
\hline Hedyotis diffusa Willd & Apoptosis & STAT3, Cyclin D1, CDK4 and Bcl-2/Bax, p21 & [49] \\
\hline DYZ-2-90 & Apoptosis & ERK, JNK & [50] \\
\hline Bufalin & Autophagy & Reactive oxygen species, JNK, ATG5, Beclin-1 & {$[53]$} \\
\hline Triterpenes & G0/G1 arrest, apoptosis, autophagy & p38 MAPK & {$[54]$} \\
\hline TLBZD formula & Cell sensecence & $\mathrm{p} 16, \mathrm{p} 21, \mathrm{RB}$ & [57] \\
\hline Norcantharidin & Metastasis & MMP-9, Sp1, NF- $\kappa$ B, STAT1 & {$[58]$} \\
\hline Berberine & cell migration & AMP-activated protein kinase, integrin $\beta 1$ & [59] \\
\hline Dauricine & $\begin{array}{c}\text { apoptosis, inhibits proliferation and } \\
\text { invasion }\end{array}$ & $\mathrm{NF}-\kappa \mathrm{B}$ & {$[60]$} \\
\hline Triptolide & Proliferation and migration & $\begin{array}{c}\text { c-myc, and A, B, C, and D-type cyclins, VEGF, } \\
\text { COX-2, thrombin receptor, CXCR4, TNF receptors, } \\
\text { and TGF- } \beta \text { receptors }\end{array}$ & {$[61]$} \\
\hline Taiwanofungus camphoratus & $\begin{array}{c}\text { Apoptosis, enhances cytotoxic effects of } \\
\text { amphotericin B }\end{array}$ & & {$[63]$} \\
\hline Tetrandrine & Apoptosis, synergize 5-Fu & $\beta$-catenin & {$[64]$} \\
\hline Notoginseng & $\begin{array}{l}\text { Enhances anti-cancer effect of 5-Fu, } \\
\text { Apoptosis }\end{array}$ & & {$[65]$} \\
\hline Adlay bran & Carcinogenesis & & {$[66]$} \\
\hline Cistanche deserticola & Carcinogenesis & $\begin{array}{c}\text { Splenic macrophage and natural killer cells, Nitric } \\
\text { oxide synthase II }\end{array}$ & [67] \\
\hline Triptolide & $\begin{array}{l}\text { Carcinogenesis, proliferation, migration, } \\
\text { colony formation, G0/G1 arrest }\end{array}$ & IL6, JAK1, IL6R, STAT3, Rac1, cyclin D1, CDK4 & {$[68]$} \\
\hline
\end{tabular}




\section{Conclusion}

TCM has certain advantages in aspects of reducing side effects and enhancing the curative effects of chemotherapy, palliating clinical syndrome, preventing recurrence and metastasis, improving quality of life and immune function, and prolonging survival time in colorectal cancer. However, with the progress of colorectal cancer treatment mode, TCM theories and studies on typing of syndrome differentiation are obviously lagging behind. TCM study on colorectal cancer should fit with the actual clinical practice to give full play to TCM advantages so as to improve clinical curative effects. Anti-cancer herb studies on colorectal cancer indicate that anti-cancer effects of TCM and chemotherapy, targeted therapy and other drug treatments share common biological basis, such as cell apoptosis, cell senescence and autophagy, etc. Current studies still cannot reveal the TCM property of anti-cancer herbs. We need to further study the action basis and compatibility of anti-cancer herbs so as to improve the curative effect of TCM treatment for colorectal cancer.

\section{Acknowledgements}

This work was partially supported by National Natural Science Foundation of China (81273726), Program from Science \& Technology Commission of Shanghai Municipality (11495801300), Three-year Action Program of Shanghai Municipality for Traditional Chinese Medicine (ZYSNXD-CC-ZDYJ017), Long-Yi Scholars and Research Team Program from State Clinical Research Center of TCM in Longhua Hospital (LYTD-04), Xing-Lin Scholars Program and Key Discipline of State Administration of Traditional Chinese Medicine (Traditional Chinese Medicine in Oncology, LHZLK-1107).

\section{REFERENCES}

[1] A. Jemal, F. Bray, M. M. Center, J. Ferlay, E. Ward and D. Forman, "Global Cancer Statistics," CA: A Cancer Journal for Clinicians, Vol. 61, No. 2, 2011, pp. 69-90. doi:10.3322/caac.20107

[2] X. R. Wang, X. B. Yuan, P. Li and L. Gao, "The TCM Syndrome Distribution in Colorectal Cancer [Chinese]," Journal of Changchun College of Traditional Chinese Medicine, 2011, Vol. 27, No. 3, 2011, pp. 377-379.

[3] S. Y. Lin, M. H. Shen, J. Lan and J. N. Shu, “Correlation of TCM Syndrome and Life Span in 780 Colon Cancer Patients [Chinese]," Journal of Traditional Chinese Medicine, Vol. 53, No. 5, 2012, pp. 410-412.

[4] G. X. Zhao, J. Zheng, Y. Gu and Y. J. Zhu, "Literature Analysis of TCM Syndrome in Colorectal Cancer [Chinese]," Journal of Liaoning University of Traditional Chinese Medicine, Vol. 11, No. 6, 2009, pp. 72-74.

[5] Z. H. Deng and J. Sun, "The Literature Study of Colorec- tal Cancer in TCM [Chinese],” Journal of Yunnan University of Traditional Chinese Medicine, Vol. 34, No. 2, 2011, pp. 54-56.

[6] X. Q. Jia, H. Z. Qiu, N. J. Huang and G. L. Lin, “A Prospective Study about Colorectal Cancer Syndrome Differentiation and Tumor Invasion and Metastasis [Chinese]," China Journal of Traditional Chinese Medicine and Pharmacy, Vol. 20, No. 6, 2005, pp. 344-346.

[7] H. Y. Zhao, D. J. Tu, X. Xia and Y. Guo, "Study on TCM Syndrome of Colorectal Carcinoma in Adjuvant Therapy Period on 151 Cases [Chinese]," China Journal of Traditional Chinese Medicine and Pharmacy, Vol. 26, No. 4, 2011, pp. 861-864.

[8] X. F. Wang, G. D. Li, S. M. Ma, X. Jiao, Z. T. Yin, H. Zhao, J. Y. Hao, D. N. Huang, Z. G. Wang, Y. Yao, X. Q. Li, H. Ren and H. S. Li, "The Study of Distribution, Composition Characteristic and the Rule of Evolution and the Correlation with Pathological Stage of TCM Syndromes of Perioperative Large Intestinal Cancer [Chinese]," Beijing Journal of Traditional Chinese Medicine, No. 8, 2010, pp. 580-582.

[9] D. J. Tu, H. Y. Zhao, J. Z. Gu, W. H. Yang and Y. Guo, "Clinical Research on the Changes of TCM Syndroms of 75 Patients with Colorectal Cancer before and after Chemotherapy [Chinese]," Chinese Archives of Traditional Chinese Medicine, Vol. 27, No. 8, 2009, pp. 1678-1680.

[10] C. Y. Xu, L. N. Li, D. M. Pan and Z. H. Wen, "Retrospective Analysis of TCM Syndrome Types Associated with Adverse Reactions after Chemotherapy Who were Advanced Large Intestine Cancer [Chinese]," Journal of Liaoning University of Traditional Chinese Medicine, Vol. 13, No. 9, 2011, pp. 104-106.

[11] J. A. Liu and Y. H. Zhang, "Effect of Pi-Shen Decoction on the Postoperative with Adverse Reaction of Chemotherapy of 96 Patients with Colorectal Cancer [Chinese]," Hunan Journal of Traditional Chinese Medicine, Vol. 16, No. 2, 2000, pp. 9-10.

[12] T. S. Mok, W. Yeo, P. J. Johnson, P. Hui, W. M. Ho, K. C. Lam, M. Xu, K. Chak, A. Chan, H. Wong, F. Mo and B. Zee, “A Double-Blind Placebo-Controlled Randomized Study of Chinese Herbal Medicine as Complementary Therapy for Reduction of Chemotherapy-Induced Toxicity," Annals Oncology: Official Journal of the European Society for Medical Oncology/ESMO, Vol. 18, No. 4, 2007, pp. 768-774.

[13] B. Cao, S. T. Li, Z. Li and W. L. Deng, "Yi-Qi-Zhu-Yu Decoction Combined with FOLFOX-4 as First-Line Therapy in Metastatic Colorectal Cancer," Chinese Journal of Integrative Medicine, Vol. 17, No. 8, 2011, pp. 593-599. doi:10.1007/s11655-011-0822-Z

[14] L. Tao, Y. J. Zhu, X. M. Lu, Y. Gu, A. G. Zhao, J. Zheng, C. G. Fu and J. K. Yang, "Clinical Study on Survival Benefit for Elderly Patients with Resected Stage II or III Colorectal Cancer Based on Traditional Chinese Medicine Syndrome Differentiation and Treatment," Zhong Xi Yi Jie He Xue Bao, Vol. 8, No. 12, 2010, pp. 115911115964. doi:10.3736/jcim20101208

[15] R. H. Huang, F. Chen, G. Q. Lao, Z. Y. Shi, J. H. Luo, D. R. Liang and J. J. Chen, "Effect of Jian-Pi-Jie-Du Decoc- 
tion on the Life Quality of Colorectal Cancer Patients in Advanced Stage [Chinese]," Hebei Journal of Traditional Chinese Medicine, Vol. 33, No. 4, 2011, pp. 494-496.

[16] Q. C. Liu, D. C. Liu, L. Song, Q. Huang, X. B. Tan and H. Zhong, "Jian-Pi-Yi-Qi Herbs Combined with Chemotherapy in the Treatment for 47 Cases with Advanced [Chinese],” Journal of Oncology, Vol. 15, No. 7, 2009, pp. 665-666.

[17] G. Wu, G. Yu, J. Li and F. Xiong, "Short Term Therapeutic Effect on Treatment of Postoperational Large Intestine Carcinoma by Fu-Pi-Yi-Wei Decoction Combined with Chemotherapy and Its Effect on Immune Function [Chinese]," Zhongguo Zhong Yao Za Zhi, Vol. 35, No. 6, 2010, pp. 782-785.

[18] J. Ma, G. J. Wang, D. F. Cai, Y. Fan and X. X. Gu, "Clinical Observation of Jian-Pi-Xiao-Liu Decoction in Preventing Postoperative Colorectal Carcinoma Recurrence and Metastasis [Chinese]," Shanghai Journal of Traditional Chinese medicine, Vol. 39, No. 1, 2005, pp. 24-25.

[19] L. Luo, Y. F. Yang, P. H. Li, Y. Wu, J. W. Zhang, X. W. Wu, S. L. Gao and D. Q. Liu, "Cohort Study on Fu-Zheng Capsule and Qu-Xie Capsule in Reducing Relapse and Metastasis of Cancer in Patients with Stage Ò and Ó Colorectal Carcinoma after Operation [Chinese],” Chinese Journal of Integrated Traditional and Western Medicine, Vol. 26, No. 8, 2006, pp. 677-680.

[20] Y. F. Yang, J. Z. Ge, Y. Wu, Y. Xu, B. Y. Liang, L. Luo, X. W. Wu, D. Q. Liu, X. Zhang, F. X. Song and Z. Y. Geng, "Cohort Study on the Effect of a Combined Treatment of Traditional Chinese Medicine and Western Medicine on the Relapse and Metastasis of 222 Patients with Stage II and III Colorectal Cancer after Radical Operation," Chinese Journal of Integrative Medicine, Vol. 14, No. 4, 2008, pp. 251-256. doi:10.1007/s11655-008-0251-9

[21] M. N. Xiong and L. G. Xiong, "Clinical Observation of Traditional Chinese Medicine Method-Yi-Qi-Jie-Du Combined with Intravenous Drip in Treatment of Advanced Colorectal Cancer [Chinese]," Modern Oncology, Vol. 18, No. 9, 2010, pp. 1839-1840.

[22] J. L. You and L. Y. Zhou, "Clinical Observation of Zhao's No. Three Decoction in the Treatment of 105 Patients with Colorectal Cancer [Chinese]," Liaoning Journal of Traditional Chinese Medicine, Vol. 36, No. 8, 2008, pp. 1201-1202.

[23] X. L. Yin, F. C. Zhao, G. Cai and Y. Li, "Clinical Observation of Spleen-Nourishing Herbs and Chemotherapy in Treating Advanced Colorectal Cancer [Chinese]," Shanghai Journal of Traditional Chinese Medicine, Vol. 45, No. 7, 2011, pp. 43-44.

[24] B. Z. Hou, X. C. Shu, S. P. Zhou, S. L. Fang and X. L. Li, "Effect of Ai-Di Injection on Basal Immunity Function of Post-Operative Patient with Carcinoma of Colon [Chinese]," Journal of Fourth Military Medical University, Vol. 29, No. 10, 2008, pp. 933-935.

[25] M. Zhang, "Ai-Di Injection in Combination with FOLFOX4 Regimen for Treatment of Advanced Colorectal Cancer [Chinese]," Chinese Journal of Clinical Oncology and
Rehabilitation, Vol. 16, No. 2, 2009, pp. 170-174.

[26] H. J. Li, L. Dong, S. Y. Fu and Y. Li, "Comparative Study on Treatment of Advanced Colorectal Cancer by Ai-Di Injection Combined with FOLFOX4 Regimen and by FOLFOX4 Regimen Alone [Chinese]," Chinese Journal of Integrative Medicine, Vol. 27, No. 12, 2007, pp. 1086-1089.

[27] L. Huang, B. Li, Z. W. Hu and P. Z. Guo, “ Effects of Matrine Injection on Immune Functions in Patients with Colorectal Carcinoma Undergoing Chemotherapy after Surgery [Chinese]," Chinese Journal of New Drugs, Vol. 20, No. 6, 2011, pp. 526-528.

[28] Q. Shi, Z. X. Wang and Q. H. Zhao, “Compound Matrine Injection Combined with FOLFIRI Chemotherapy in the Treatment of 50 Patients with Advanced Colorectal Cancer [Chinese]," Chinese Journal of New Drugs, Vol. 19, No. 17, 2010, pp. 1589-1592.

[29] D. Long, Y. X. Zhao, Z. Ma, Z. Z. Liu and Q. X. Tan, "Compound Matrine Injection in the Improvement of Life Quality of Patients with Advanced Colorectal Carcinoma in Different Types of Syndromes: A Clinical Study [Chinese]," Evaluation and Analysis of Drug-Use in Hospitals of China, Vol. 10, No. 6, 2010, pp. 552-553.

[30] B. Xiao, S. D. Cui, C. L. Chen, W. Guo, B. P. Wu, Y. L. Yao, Y. Bai, S. D. Liu, J. D. Wang, T. M. Cheng, L. Lan, Y. L. Zhang, F. C. Zhi, Z. S. Zhang, W. D. Zhang and B. Jiang, "Effect of Chinese Patent Medicine Combined with Chemotherapy on Treating Patients with Colorectal Cancer [Chinese]," Chinese Journal of Integrated Traditional and Western Medicine on Digestion, Vol. 16 No. 3, 2008, pp. 178-181.

[31] X. R. Jing, J. Chen, M. Min, G. C. Zhong, L. L. Yue, H. Liao and Y. R. Wang, "Clinical Efficacy of Shen-QiFu-Zheng Injection Combined with Chemotherapy on Advanced Colorectal Carcinoma [Chinese]," Modern Oncology, Vol. 115, No. 13, 2007, pp. 382-384.

[32] Q. L. Liang, D. C. Pan, J. R. Xie, L. Z. Cai, S. J. Li and Q. Yang, "Effect of Shen-Qi-Fu-Zheng Injection Combined with Chemotherapy in Treating Advanced Colorectal Carcinoma [Chinese]," Chinese Journal of Integrated Traditional and Western Medicine, Vol. 29, No. 5, 2009, pp. 439-441.

[33] M. Yuan, G. L. Huang, Z. G. Li and W. Ding, "Effect of Combined Kui-Er Granule and Chemotherapy on Immunity in Patients with Colorectal Carcinoma Postoperation [Chinese]," China Cancer, Vol. 14, No. 7, 2005, pp. 487-488.

[34] Y. J. Hai, H. B. Zhou, Y. Zheng, G. K. Zhang, M. Wang, L. Lu, Z. Q. Wang, X. F. Zhao, H. J. Cui and Z. Y. Hao, "Clinical Effect of Kui-Er Granules in Advanced Colorectal Cancer Patients [Chinese]," China Pharmacist, Vol. 15, No. 4, 2012, pp. 519-521.

[35] X. L. Ling and D. Zhao, "Short Term Efficacy of Combination of Oxaliplatin, Capecitabine and Kang-Lai-Te in the Treatment of Advanced Colorectal Carcinoma [Chinese]," Medical Information, Vol. 24, No. 2, 2011, pp. 377-379.

[36] J. H. Ran, J. H. Zhang, X. Wang, Q. Guo and J. Yang, "Effect of Kang-Lai-Te on Immunologic Function of 
Postoperative Patients with Large Intestine Cancer [Chinese]," Chinese Journal of Clinical Oncology and Rehabilitation, Vol. 6, No. 2, 1999, pp. 20-21.

[37] K. P. Shen, B. Hu, H. Zhang, H. Zhou, X. Gu, C. F. Pan, L. M. Zhu and G. X. Ruan, "Effect of Astragalus Injection Combined with IL-2 to Improve the Immune Function of Patients with Colorectal Carcinoma [Chinese]," Pharmacology and Clinics of Chinese Materia Medica, Vol. 24, No. 2, 2008, pp. 99-101.

[38] J. L. Lv, J. Y. Sun, N. R. Yu, Y. Yuan, Z. J. Lu and H. Y. Liu, "Effect of Astragalus Polysaccharide on the Prevention of Leukocyte Reduction before Chemotherapy of Colorectal Cancer [Chinese]," Journal of Chinese Medicinal Materials, Vol. 32, No. 1, 2009, pp. 166-168.

[39] J. Portugal, M. Bataller and S. Mansilla, "Cell Death Pathways in Response to Antitumor Therapy," Tumori, Vol. 95, No. 4, 2009, pp. 409-421.

[40] M. V. Chiantore, S. Vannucchi, G. Mangino, Z. A. Percario, E. Affabris, G. Fiorucci and G. Romeo, "Senescence and Cell Death Pathways and Their Role in Cancer Therapeutic Outcome," Current Medicinal Chemistry, Vol. 16, No. 3, 2009, pp. 287-300. doi:10.2174/092986709787002691

[41] J. M. Coates, J. M. Galante and R. J. Bold, "Cancer Therapy Beyond Apoptosis: Autophagy and Anoikis as Mechanisms of Cell Death,” The Journal of Surgical Research, Vol. 164, No. 2, 2010, pp. 301-308. doi:10.1016/j.jss.2009.07.011

[42] B. Hu, K.-P. Shen, H.-M. An, Y. Wu and Q. Du, “Aqueous Extract of Curcuma aromatica Induces Apoptosis and G2/M Arrest in Human Colon Carcinoma LS-174-T Cells Independent of P53," Cancer Biotherapy and Radiopharmaceuticals, Vol. 26, No. 1, 2011, pp. 97-104. doi:10.1089/cbr.2010.0853

[43] W. W. Huang, S. W. Ko, H. Y. Tsai, J. G. Chung, J. H. Chiang, K. T. Chen, Y. C. Chen, H. Y. Chen, Y. F. Chen and J. S. Yang, "Cantharidin Induces G2/M Phase Arrest and Apoptosis in Human Colorectal Cancer Colo 205 Cells through Inhibition of CDK1 Activity and Caspase-Dependent Signaling Pathways," International Journal of Oncology, Vol. 38, No. 4, 2011, pp. 1067-1073.

[44] Z. Y. Wang, D. M. Wang, T. Y. Loo, Z. Y. Wang, D. M. Wang, T. Y. Loo, Y. Cheng, L. L. Chen, J. G. Shen, D. P. Yang, L. W. Chow, X. Y. Guan and J. P. Chen, "Spatholobus Suberectus Inhibits Cancer Cell Growth by Inducing Apoptosis and Arresting Cell Cycle at G2/M Checkpoint,” Journal of Ethnopharmacology, Vol. 133, No. 2, 2011, pp. 751-758. doi:10.1016/j.jep.2010.11.004

[45] K. C. Lai, Y. J. Chiu , Y. J. Tang, K. L. Lin, J. H. Chiang, Y. L. Jiang, H. F. Jen, Y. H. Kuo, S. Agamaya, J. G. Chung and J. S. Yang, "Houttuynia cordata Thunb Extract Inhibits Cell Growth and Induces Apoptosis in Human Primary Colorectal Cancer Cells," Anticancer Research, Vol. 30, No. 9, 2010, pp. 3549-3556.

[46] J. Lin, Y. Chen, L. Wei, X. Chen, W. Xu, Z. Hong, T. J. Sferra and J. Peng, "Hedyotis diffusa Willd Extract Induces Apoptosis via Activation of the Mitochondri-on-Dependent Pathway in Human Colon Carcinoma Cells,” International Journal of Oncology, Vol. 37, No. 5,
2010, pp. 1331-1338.

[47] V. Milacic, S. Banerjee, K. R. Landis-Piwowar, F. H. Sarkar, A. P. Majumdar and Q. P. Dou, "Curcumin Inhibits the Proteasome Activity in Human Colon Cancer Cells in Vitro and in Vivo," Cancer Research, Vol. 68, No. 18, 2008, pp. 7283-7292. doi:10.1158/0008-5472.CAN-07-6246

[48] X. L. Tang, X. Y. Yang, Y. C. Kim, S. Y. Kim, B. D. Kang, D. Y. Choi, O. J. Kim, W. C. Park and H. Park, "Protective Effects of the Ethanolic Extract of Melia Toosendan Fruit against Colon Cancer," Indian Journal of Biochemistry \& Biophysics, Vol. 49, No. 3, 2012, pp. 173-181.

[49] Q. Cai, J. Lin, L. Wei, L. Zhang, L. Wang, Y. Zhan, J. Zeng, W. Xu, A. Shen, Z. Hong and J. Peng, " Hedyotis diffusa Willd Inhibits Colorectal Cancer Growth in Vivo via Inhibition of STAT3 Signaling Pathway," International Journal of Molecular Science, Vol. 13, No. 5, 2012, pp. 6117-6128. doi:10.3390/ijms13056117

[50] L. T. Wang, S. L. Pan, T. H. Chen, Y. Dong, K. H. Lee and C. M. Teng, "DYZ-2-90, a Novel Neo-Tanshinlactone Ring-Opened Compound, Induces ERK-Mediated Mitotic Arrest and Subsequent Apoptosis by Activating JNK in Human Colorectal Cancer Cells," Chembiochem: A European Journal of Chemical Biology, Vol. 13, No. 11, 2012, pp. 1663-1672. doi:10.1002/cbic.201200191

[51] H. M. Lien, H. W. Lin, Y. J. Wang, L. C. Chen, D. Y. Yang, Y. Y. Lai and Y. S. Ho, "Inhibition of Anchorage-Independent Proliferation and G0/G1 Cell-Cycle Regulation in Human Colorectal Carcinoma Cells by 4,7-Dimethoxy-5-Methyl-1,3-Benzodioxole Isolated from the Fruiting Body of Antrodia Camphorate," EvidenceBased Complementary and Alternative Medicine: ECAM, Vol. 2011.

[52] P. Maycotte and A. Thorburn, "Autophagy and Cancer Therapy,” Cancer Biology \& Therapy, Vol. 11, No. 2, 2011, pp. 127-137. doi:10.4161/cbt.11.2.14627

[53] C. M. Xie, W. Y. Chan, S. Yu, J. Zhao and C. H. Cheng, "Bufalin Induces Autophagy-Mediated Cell Death in Human Colon Cancer Cells through Reactive Oxygen Species Generation and JNK Activation," Free Radical Biology \& Medicine, Vol. 51, No. 7, 2011, pp. 1365-1375. doi:10.1016/j.freeradbiomed.2011.06.016

[54] A. Thyagarajan, A. Jedinak, H. Nguyen, C. Terry, L. A. Baldridge, J. Jiang and D. Sliva, “Triterpenes from Ganoderma Lucidum Induce Autophagy in Colon Cancer through the Inhibition of p38 Mitogen-Activated Kinase (p38 MAPK)," Nutrition Cancer, Vol. 62, No. 5, 2010, pp. 630-640. doi:10.1080/01635580903532390

[55] B. Hu, H. M. An and K. P. Shen, "Cellular Senescence and Carcinogenesis," Chinese Bulletin of Life Sciences, Vol. 20, No. 3, 2008, pp. 447-449.

[56] J. A. Ewald, J. A. Desotelle, G. Wildin and D. F. Jarrard, "Therapy-Induced Senescence in Cancer," Journal of $\mathrm{Na}$ tional Cancer Institute, Vol. 102, No. 20, 2010, pp. 15361546. doi:10.1093/jnci/djq364

[57] B. Hu, H. M. An, K. P. Shen and Q. Du, "SenescenceInducing Effects of Chinese Herbal Medicine Tenglong Buzhong Decoction on Human Colon Carcinoma LS- 
174-T Cells and the Mechanism,” Journal of Chinese Integrative Medicine, Vol. 8, No. 11, 2010, pp. 1048-1052. doi:10.3736/jcim20101108

[58] Y. J. Chen, W. M. Chang, Y. W. Liu, C. Y. Lee, Y. H. Jang, C. D. Kuo and H. F. Liao, “ A Small-Molecule Metastasis Inhibitor, Norcantharidin, Downregulates Matrix Metalloproteinase-9 Expression by Inhibiting Sp1 Transcriptional Activity in Colorectal Cancer Cells," Chemico-Biological Interactions, Vol. 181, No. 3, 2009, pp. 440-446. doi:10.1016/j.cbi.2009.07.004

[59] J. J. Park, S. M. Seo, E. J. Kim, Y. J. Lee, Y. G. Ko, J. Ha and M. Lee, "Berberine Inhibits Human Colon Cancer Cell Migration via AMP-Activated Protein Kinase-mediated Downregulation of Integrin $\beta 1$ signaling," Biochemical and Biophysical Research Communications, Vol. 426, No. 4, 2012, pp. 461-467. doi:10.1016/j.bbrc.2012.08.091

[60] Z. Yang, C. Li, X. Wang, C. Zhai, Z. Yi, L. Wang, B. Liu, B. Du, H. Wu, X. Guo, M. Liu, D. Li and J. Luo, "Dauricine Induces Apoptosis, Inhibits Proliferation and Invasion through Inhibiting NF- $k$ B Signaling Pathway in Colon Cancer Cells," Journal of Cellular Physiology, Vol. 225, No. 1, 2010, pp. 266-725. doi:10.1002/jcp.22261

[61] S. M. Johnson, X. Wang and B. M. Evers, "Triptolide Inhibits Proliferation and Migration of Colon Cancer Cells by Inhibition of Cell Cycle Regulators and Cytokine Receptors,” Journal of Surgical Research, Vol. 168, No. 2, 2011, pp.197-205. doi:10.1016/j.jss.2009.07.002

[62] Z. He, D. Subramaniam, S. Ramalingam, A. Dhar, R. G. Postier, S. Umar, Y. Zhang and S. Anant, "Honokiol Radiosensitizes Colorectal Cancer Cells: Enhanced Activity in Cells with Mismatch Repair Defects," American Journal of Physiology Gastrointestinal and Liver Physiology, Vol. 301, No. 5, 2011, pp. G929-G937. doi:10.1152/ajpgi.00159.2011

[63] L.Y. Chen, M. T. Sheu , D. Z. Liu, C. K. Liao, H. O. Ho,
W. Y. Kao, Y. S. Ho, W. S. Lee and C. H. Su, "Pretreatment with an Ethanolic Extract of Taiwanofungus Camphoratus (Antrodia camphorata) Enhances the Cytotoxic Effects of Amphotericin B," Journal of Agricultural and Food Chemistry, Vol. 59, No. 20, 2011, pp. 11255-11263. doi:10.1021/jf2024215

[64] B. C. He, J. L. Gao, B. Q. Zhang, Q. Luo, Q. Shi, S. H. Kim, E. Huang, Y. Gao, K. Yang, E. R. Wagner, L. Wang, N. Tang, J. Luo, X. Liu, M. Li, Y. Bi, J. Shen, G. Luther, N. Hu, Q. Zhou, H. H. Luu, R. C. Haydon, Y. Zhao and T. C. He, "Tetrandrine Inhibits Wnt $/ \beta$-catenin Signaling and Suppresses Tumor Growth of Human Colorectal Cancer," Molecular Pharmacology, Vol. 79, No. 2, 2011, pp. 211-219. doi:10.1124/mol.110.068668

[65] C. Z. Wang, X. Luo , B. Zhang, W. X. Song, M. Ni , S. Mehendale, J. T. Xie , H. H. Aung, T. C. He and C. S. Yuan, "Notoginseng Enhances Anti-Cancer Effect of 5Fluorouracil on Human Colorectal Cancer Cells," Cancer Chemotherapy and Pharmacology, Vol. 60, No. 1, 2007, pp. 69-79. doi:10.1007/s00280-006-0350-2

[66] S. C. Li, C. M. Chen, S. H. Lin, W. Chiang and C. K. Shih, "Effects of Adlay Bran and Its Ethanolic Extract and Residue on Preneoplastic Lesions of the Colon in Rats," Journal of the Science of Food and Agriculture, Vol. 91, No. 3, 2011, pp. 547-552. doi:10.1002/jsfa.4219

[67] Y. Jia, Q. Guan, Y. Guo and Du C, "Reduction of Inflammatory Hyperplasia in the Intestine in Colon Cancer-Prone Mice by Water-Extract of Cistanche deserticola,” Phytotherapy Research: PTR, Vol. 26, No. 6, 2012, pp. 812-819.

[68] Z. Wang, H. Jin, R. Xu, Q. Mei and D. Fan, “Triptolide Downregulates Rac1 and the JAK/STAT3 Pathway and Inhibits Colitis-Related Colon Cancer Progression,” Experimental \& Molecular Medicine, Vol. 41, No. 10, 2009, pp. 717-727. doi:10.3858/emm.2009.41.10.078 